\title{
ANALISE DA PSICOLOGIA DO ESPORTE EM PERIÓDICOS DE LÍNGUA INGLESA
}

Simone Salvador Gomes

Danilo Reis Coimbra

Renato Miranda

Mauricio Bara Filho

\section{Resumo}

O objetivo do estudo foi analisar a psicologia do esporte nos periódicos de língua inglesa. Foram selecionados 521 resumos (2002 a 2006), de quatro periódicos. Analisaram-se os temas, amostra e o tipo de estudo utilizado. A amostra mais pesquisada foi de atletas de esporte competitivo $(57,2 \%)$, seguido por estudos com não atletas $(29,3 \%)$ e atletas juvenis (12,5\%). Em relação ao tipo de estudo, os de revisão tiveram $15,8 \%$, intervenção $15,4 \%$ e desenvolvimento de instrumentos $11 \%$, observação e descrição de um fenômeno psicológico obteve $57,9 \%$. Os temas mais pesquisados foram: motivação $(15,7 \%)$, capacidade visual e mental $(11,9 \%)$, humor $(11,7 \%)$ e auto-eficácia $(10,6 \%)$. São necessários estudos que verifiquem diferenças e semelhanças entre os resultados deste estudo e as pesquisas brasileiras.

\section{Palavras-Chave}

Análise; Psicologia do Esporte; Língua inglesa.

\section{IT ANALYZES OF THE PSYCHOLOGY OF THE SPORT IN PERIODIC OF ENGLISH LANGUAGE.}

Simone Salvador Gomes

Danilo Reis Coimbra

Renato Miranda,

Mauricio Bara Filho

\begin{abstract}
The purpose of the study was to analyze the scientific researches on Sport Psychology in journals published in English. 521 summaries were selected (2002 to 2006), of four journals. The analyzed were: research topics, sample type and study method. The sample more researched was athletes to competitive sport $(57,2 \%)$, following for studies with non-athletes $(29,3 \%)$ and juvenile athletes $(12,5 \%)$. in relation to the study type, revision $15,8 \%$, intervention $15,4 \%$ and development instruments $11 \%$, observation and description to psychological phenomenon obtained $57,9 \%$. The themes more researched were: motivation $(15,7 \%)$, visual and mental capacity $(11,9 \%)$, poms $(11,7 \%)$ and self concept $(10,6 \%)$. Necessary studies that verify differences and similarities between the results of this study and the Brazilian researches.
\end{abstract}

\section{Key-Words}

Analysis; Sport psychology; English language. 


\section{INTRODUÇÃO}

O esporte contemporâneo é considerado um dos maiores fenômenos sociais do século XX. O atleta tem que apresentar o máximo de rendimento com a maior rapidez possível, a performance de hoje não é o suficiente amanhã, pois se exige que ele ultrapasse seus limites a cada competição (Sanzano, 2003). Esses atletas necessitam ser preparados por uma gama de profissionais que estão sendo inseridos neste contexto, agregando um grande número de áreas afins que constituem as chamadas Ciências do Esporte, e incluem a Medicina Desportiva, a Nutrição Esportiva, a Biomecânica, a Sociologia, a Psicologia do Esporte entre outras, com o objetivo de aperfeiçoar a performance do atleta ou aprimorar a saúde e a qualidade de vida dos indivíduos através da prática de atividades físicas (SOUSA FILHO, 2000; BARA FILHO; MIRANDA, 1998; DE ROSE JUNIOR, 2000; WEINBERG; GOULD, 2001; SAMULSKI, 2002; DOSIL, 2004).

A Psicologia do Esporte é a disciplina acadêmica que tem como objeto de estudo as diferentes dimensões psicológicas da conduta humana no contexto do esporte e da atividade física. Investiga as causas e os efeitos de ocorrências psíquicas que o ser humano apresenta antes, durante e após o exercício, sendo estes educativos, recreativos, competitivos ou reabilitador (BECKER JR., 1995).

O principal objetivo da Psicologia do Esporte é entender a influência dos fatores psicológicos no desempenho físico de um indivíduo e como a participação em esportes e exercício afeta o desenvolvimento psicológico, a saúde e o bem estar de uma pessoa.

De acordo com Weinberg; Gould (2001), a relação entre psicologia e esporte teve inicio no final do século XIX quando Norman Triplett, um psicólogo, queria entender por que os ciclistas pedalavam mais rápido quando em grupos, do que sozinhos. Anos depois, quando Coleman Griffith, desenvolveu o primeiro laboratório em psicologia do esporte, essa relação passou a ser melhor definida. Surgiram novos centros de pesquisas em todo o mundo, melhorando a quantidade e qualidade das publicações na área. E, finalmente, quando em 1965, realizou-se em Roma o Primeiro Congresso Mundial de Psicologia do Esporte, esta disciplina passou a ser definida como uma Ciência do Esporte.

A partir de 1978 houve um enorme crescimento da psicologia do esporte, principalmente da área aplicada. Ela atinge um topo nas pesquisas, com instrumentos adequados, situações e populações específicas; muitos laboratórios especializados, universidades e faculdades com departamentos de Psicologia do Esporte e numerosa literatura de nível científico e revistas especializadas de bom nível. 
Observa-se um aumento significativo dos estudos, trabalhos, artigos e publicações em Psicologia do Esporte devido a um maior número de pesquisadores interessados na área, tanto psicólogos, quanto profissionais da área do esporte. Devido a esse aumento considerável de pesquisas, são necessários novos estudos que verifiquem os temas comumente abordados na área, identificando os mais investigados e sua evolução, assim como aqueles que necessitam de uma maior atenção por parte dos pesquisadores. Deste modo, analisando a produção cientifica na Psicologia do Esporte nacional e internacional, podemos nortear as diretrizes, identificar futuras tendências de pesquisas e suas características, para que futuramente seja feita uma interface do que está sendo investigado pelos psicólogos do esporte e a sua aplicação na prática esportiva.

Isto posto, o objetivo do presente estudo foi analisar a produção cientifica sobre Psicologia do Esporte nos periódicos de língua inglesa, pois estes são uma das principais fontes de consulta dos pesquisadores do mundo inteiro e onde a Psicologia do Esporte j á está consolidada.

\section{MÉTODO}

Para a análise selecionamos como amostra 521 resumos dos artigos publicados entre 2002 e 2006, dos seguintes Periódicos de língua inglesa: Journal of Sport and Excercise Psychology, The Sport Psychologist, International Journal of Sport Psychology, Psychology of Sport and Excercise.

No presente estudo foram analisados os seguintes aspectos:

- Temas pesquisados. Foram 21 temas pertinentes da $\mathrm{P}$ selecionados $\mathrm{e}$ classificados a partir da consulta feita na literatura especifica de psicologia do esporte e a partir da própria análise.

- Tipo de amostra utilizada. Atletas de nível competitivo, atletas juvenis que participam de competição e indivíduos praticantes de atividade física, que não são atletas.

- Tipo de estudo. Os métodos de investigação utilizados nas pesquisas, os de revisão e discussão de um tema, intervenção, observação e descrição de um fenômeno e trabalhos de desenvolvimento de novos métodos e instrumentos.

Foi realizada uma estatística descritiva com os percentuais geral e especifico de cada item e posteriormente, um teste de Qui-quadrado para analisar a existência das diferenças estatisticamente significativas $(\mathrm{p}<0,05)$ entre as variáveis. 


\section{RESULTADOS}

Figura 1 - Amostra utilizada nos estudos

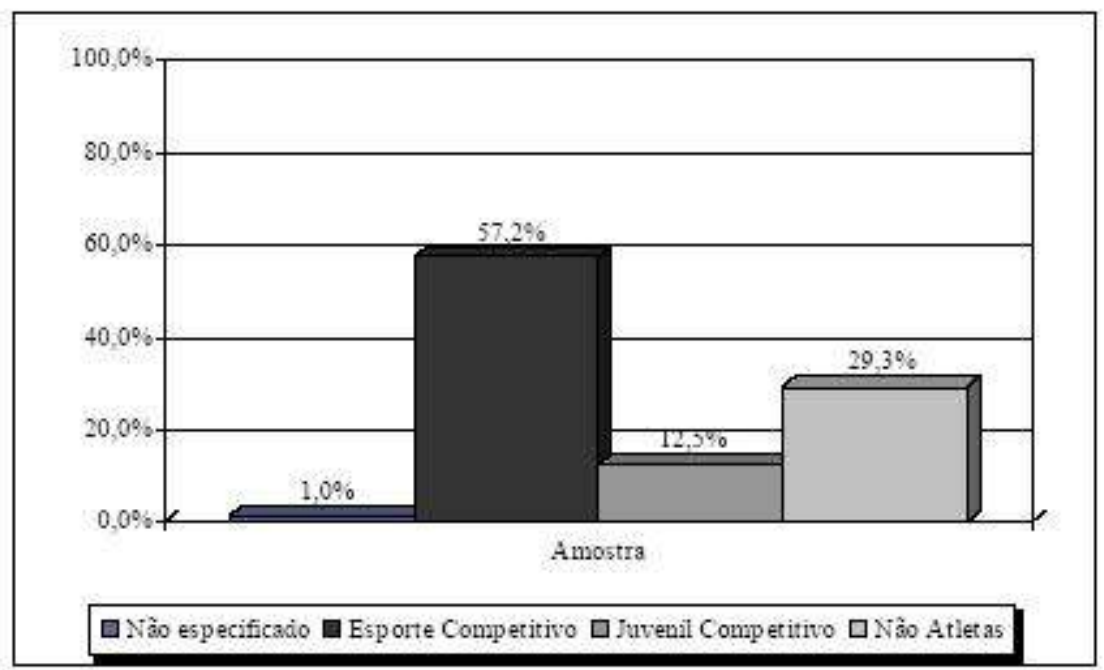

Observa-se na Figura 1 que a amostra mais utilizada nas pesquisas foi de atletas de esporte competitivo $(57,2 \%)$, seguido pelos estudos com não atletas $(29,3 \%)$ e por ultimo atletas juvenis que participam de competição (12,5\%). Os estudos que não especificaram a amostra ou abordavam temas aplicados a qualquer uma tiveram $1 \%$ dos estudos.

Figura 2 - Tipos de Estudo

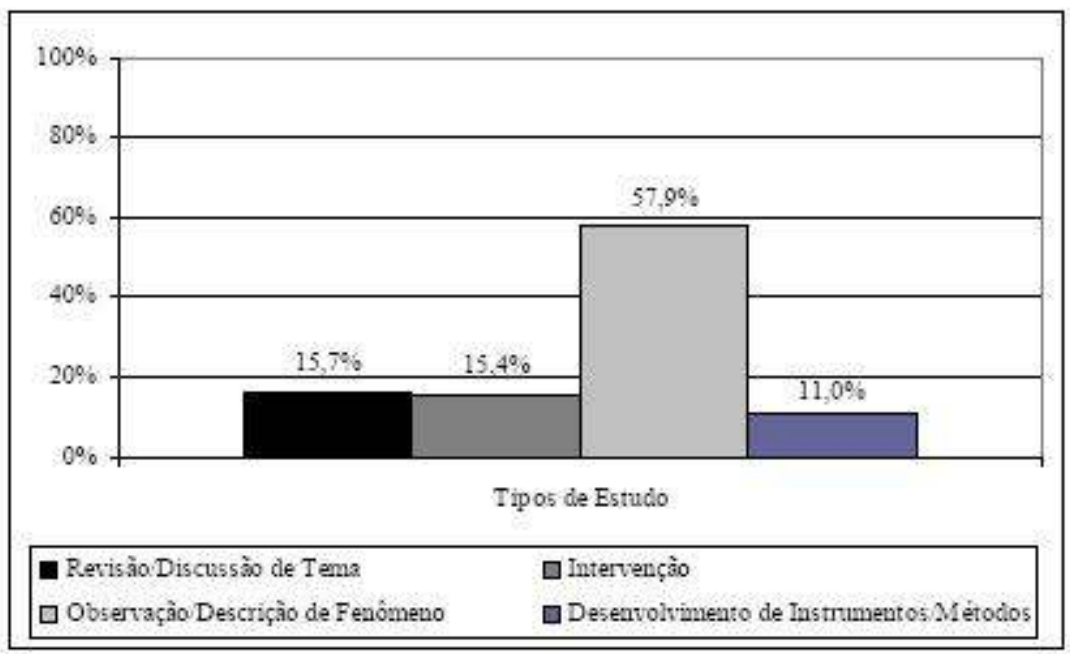


Em relação ao tipo de estudo (Figura 2), observou-se que três tipos de estudo, Revisão (15,8\%), Intervenção $(15,4 \%)$ e Desenvolvimento de instrumentos (11\%) tiveram um maior equilíbrio, enquanto que observação e descrição de um fenômeno psicológico $(57,9 \%)$ obteve mais da metade das pesquisas.

Tabela 3- Temas estudados

\begin{tabular}{|c|c|}
\hline Tema Pais & $\begin{array}{l}\text { Lingua } \\
\text { Inglesa }\end{array}$ \\
\hline 1 Motivaçào & $15,7 \%$ \\
\hline 2. Humor/Efeitos psicológicos/Afeto/Emoções & $11,7 \%$ \\
\hline 3. Situaçào de jogo. Tomada de decisào & $3,8 \%$ \\
\hline 4. Coesào Liderança & $2,9 \%$ \\
\hline 5. Ansiedade/Stress/Bunnout & $8,2 \%$ \\
\hline 6. Capacidade visual e mental/Rendimento motor/Atençào/Cogniçào & $11,9 \%$ \\
\hline 7. Auto-eficácia Confiança/magem & $10,6 \%$ \\
\hline 8. Coping & $1,7 \%$ \\
\hline 9. Perfeccionismo & $1 \%$ \\
\hline 10. Abandono & $0,4 \%$ \\
\hline 11. Carreira despontiva & $2,3 \%$ \\
\hline 12. Treinador Professor de Educaçào Fisica & $5 \%$ \\
\hline 13. Comportamento no exercicio & $4,8 \%$ \\
\hline 14. Lesão & $1,3 \%$ \\
\hline 15. Agressào & $1,2 \%$ \\
\hline 16. Psicólogo do Esporte Programas e cursos & $7,3 \%$ \\
\hline 17. Disturbios alimentares Drogas/Doping & $1,3 \%$ \\
\hline 18. Personalidade & $0.8 \%$ \\
\hline 19. Aspectos sociais & $4,6 \%$ \\
\hline 20. Efeitos psicofisiológicos & $2,9 \%$ \\
\hline 21. Caracteristicas psicológicas de exportistas/ábitros & $0,6 \%$ \\
\hline
\end{tabular}

Os temas mais encontrados nos estudos (Tabela 10) foram de Motivação (15,7\%), seguido por aqueles relacionados à área da aprendizagem motora, os de Capacidade visual e mental, rendimento motor, atenção e cognição $(11,9 \%)$. Em terceiro, os estudos sobre humor, efeitos psicológicos do exercício e emoções, com 11,7\%. Auto-eficácia, autoconfiança e auto-imagem foram encontrados em 10,6\% dos estudos.

Abandono (0,4\%), Características psicológicas de esportistas e árbitros $(0,6 \%)$, e Personalidade $(0,8 \%)$, foram os temas menos encontrados nas pesquisas.

\section{CONSIDERAÇÕES FINAIS}

A partir da análise dos resultados podemos concluir que o número de publicações nos periódicos de língua inglesa é bastante considerável, pois o número de artigos analisados no período de 5 anos (2002 a 2006) foi de 521. 
Verificou-se também certa tendência dos estudos com amostra de atletas competitivos, pois mais da metade dos trabalhos tiveram essa população. Em relação ao tipo de estudo, observou-se uma discrepância dos estudos de observação e descrição dosa fenômenos, enquanto que as intervenções psicológicas, revisão e discussão de um tema e trabalhos visando o desenvolvimento de instrumentos e métodos de aplicação, que também são muito importantes foram pouco explorado durante o período da análise (2002 a 2006). Na análise dos temas estudados pela Psicologia do Esporte, observo-se um desequilíbrio entre os temas mais estudados e os menos estudados, mostrando uma tendência dos pesquisadores por determinados temas. Enquanto os cinco temas mais estudados (motivação, capacidade visual e mental, humor, auto-eficácia e ansiedade) somados foram focos de mais da metade das pesquisas, temas como abandono, características psicológicas de esportistas e árbitros, personalidade e perfeccionismo tiveram menos de $1 \%$ das pesquisas analisadas.

Futuros estudos são necessários para verificar diferenças entre as pesquisas publicadas em países de língua inglesa e os trabalhos realizados no Brasil e em outros países onde a psicologia do esporte está segmentada a fim de observar diferenças e semelhanças com esta análise. É preciso averiguar também, se o que os pesquisadores da psicologia do esporte estão estudando é realmente a necessidade de atletas e treinadores para a prática do seu esporte.

\section{REFERÊNCIAS}

BARA FILHO, M. G.; MIRANDA, R. Aspectos psicológicos do esporte competitivo. Revista Treinamento Desportivo. v. 3, p. 62-72, 1998. . Manual de psicologia do esporte \& exercício. Porto Alegre: Nova Prova, 2000.

BURITI, M. A, WITTER, G. W, BURITI, M. S. Psicologia no The Sport Psychologist (1995/1996): análise de produção cientifica. In: BURITI, M. A. (Org.). Psicologia do esporte. Campinas: Alínea, 1997, p. 75-188.

CASTIllo, I.; AlVAREZ, O.; BALAGUER, I. Temas de Investigación sobre aspectos Psicosociales del deporte a través de la base de dados PSYCINFO (1887- 2001). Revista de Psicología del Deporte, v. 14, p.109-123; 2005

DE ROSE JR.; D. História e evolução da Psicologia do Esporte. Revista Paulista de Educação Física, v. 6, p. 73-78, 1992.

DOBRÁNSKY, I.A. Técnicos no The Sport Psychologist e Revista Paulista de Educação Física: análise da produção científica (1994/2003). Revista Movimento \& Percepção. Espírito Santo de Pinhal, 
v. 4, p. 74-86; 2004.

DOSIL, J. Psicología de la Activiade Física y del Deporte. Madrid: McGrawHill; 2004.

FONSECA, A. M. A psicologia do desporto e a batalha da qualidade. Revista Portuguesa de Ciência do Desporto. 2001. v.1, n.1, p. 114-123.

GARCÉS DE LOS FAYOS, E. J.; BENEDICTO, L. V.; DOSIL, J. Nuevas aportaciones en psicología del deporte. Una mirada crítica sobre la última década de nuestra disciplina. Cuadernos de Psicología del Deporte, v. 4, p. 7-17, 2004.

GARCIA-MAS, A. La psicologia del deporte y sus relaciones con otras ciencias del deporte. Revista de Psicología del Deporte, v. 11, p. 103-113, 1997

SAMULSKI, D. Psicologia do Esporte. Barueri: Manole, 2002.

SIMÕES, A. C.; et al. A psicossociologia como área de conhecimento da ciência do esporte. Revista Paulista de Educação Física. v. 18, p. 73-81; 2004.

SOUSA FILHO, P.G. O que é psicologia dos esportes?. Revista Brasileira de Ciência e Movimento. v. 8 , p. $33-36,2000$

WEINBERG, R. S.; GOULD, D. Fundamentos da psicologia do esporte e do exercício. 2. Porto Alegre: Artmed, 2001. 


\title{
Simone Salvador Gomes
}

Universidade Federal de Juiz de Fora

\section{Danilo Reis Coimbra}

Universidade Federal de Juiz de Fora

\author{
Renato Miranda \\ Universidade Federal de Juiz de Fora
}

Maurício Bara Filho

Universidade Federal de Juiz de Fora

\section{Referência do artigo:}

\section{ABNT}

GOMES, S. S. et al. Análise da psicologia do esporte em periódicos de língua inglesa. Conexões, v. 6, p. 430437, 2008.

\section{APA}

Gomes, S. S., Coimbra, D. R., Miranda, R., \& Filho, M. B. (2008). Análise da psicologia do esporte em periódicos de língua inglesa. Conexões, 6, 430-437.

\section{VANCOUVER}

Gomes SS, Coimbra DR, Miranda R, Filho MB. Análise da psicologia do esporte em periódicos de língua inglesa. Conexões, 2008; 6: 430-437. 